\title{
Musik - Sprache - (Sprach-)Bild. Zur Semiotizität italienischer barocker Gleichnisarien (2. Teil)
}

\author{
Marco AGNETTA (Hildesheim)
}

\section{Summary}

The aim of the present study is to describe some of the possible relations between music, language and verbal picture (or metaphor) which are given in the drammi per musica of the early 18th century and, especially, in four allegorical arias in Vivaldi's opus La Griselda (1735). This paper sketches some aspects of the cooperation between librettist (Goldoni) and composer (Vivaldi) as well as the importance of metaphor for the interlinkage of music and language in such arias. The essay is divided up into two parts: the first part, which has been presented in ATeM 2 (2017), outlines the bipartite textual structure of the mentioned simile arias (metaphoric vs. literal stanza) which recalls the same two-piece structure of renaissance and baroque emblems. The second part which is presented in the following will focus on the interaction of poetic language, verbal image and Vivaldi's music in the mentioned simile arias. Here it is explained why questions on musical semantics primarily require a theory of the sign occurrence before generalizations can be carried out on the level of sign systems. The contribution also sheds light on the central role of internal sign learning processes that may transcend arias, entire operas and other combined artworks.

\section{Semiotische Inhärenzen und Textvertonung}

Die Tätigkeit eines Librettodichters ist von vorausschauenden Maßnahmen geprägt, die sich aus der Bestimmung des von ihm zu erstellenden Endprodukts zur musikdramatischen Verwirklichung ergeben (cf. hierzu u. a. Gier 1999, 9). Aus einer semiotischen Perspektive betrachtet, bedingt ein Librettist dabei auf zweifache Weise die Vertonung des von ihm verfassten Operntextes durch einen Komponisten:

Zum einen bestimmt er durch stilistische Entscheidungen wie die Wortwahl und -stellung, die Silbenzahl, das metrische Schema, die Verteilung von Wort- und Satzakzenten sowie auch durch rhetorische Figuren etc. die Prosodie des Textes. Diese Größen, die allgemein 
vom Emittenten einer sprachlichen Botschaft bewusst (und manchmal auch unbewusst) gestaltet werden, bezeichnen wir als musikalische Inhärenzen, weil sie die Klangmaterialität der Sprache ausmachen und weil sie der Rede inhärent sind. (Wir vermuten, dass diese klanglichen Merkmale eines parole-Aktes infolge einer Abstraktion bis zu einem bestimmten Ausmaß auch auf der langue-Ebene beschrieben werden können.) Freilich sind Sprechmelodie und Sprechrhythmik durch die textlichen Auswahlprozesse nur rudimentär vorgegeben. Dies veranschaulicht gerade das Verfahren der Textvertonung, bei dem der Komponist die definitiven Tonhöhen respektive Notenwerte erst festlegt, die aus einer existierenden Textgrundlage auch ein musikalisches Werk im engeren Sinne machen. So unterentwickelt und approximativ Melos und Rhythmus als vom Textdichter festgelegte Größen also vor der Vertonung auch sind, sie müssen vom Komponisten stets als Vorgaben mitberücksichtigt werden. In instrumentalbegleiteten Gesangsstücken führen die musikalischen Inhärenzen des Librettotextes dabei nicht nur zur Festsetzung der Gesangslinie, in welche der Text eingebettet ist, sondern bedingen auch die Instrumentalstimmen, greifen diese doch oft auf melodische und rhythmische Keimzellen der Gesangspartie zurück.

Zum anderen beeinflusst ein Librettist die musikalische Realisation und Begleitung seiner Verse durch die Gestaltung der Inhaltsseite bzw. Semantik der Sprachzeichen, u. a. durch Tropen etc. Wir sprechen in diesem Kontext - um einen Terminus wiederaufzugreifen, der im ersten Teil dieses Artikels vorgestellt worden ist (cf. Agnetta 2017, 13) - von den bildlich-szenischen Inhärenzen des Librettotextes bzw. des Sprachsystems. Das Kernmerkmal eines guten Librettos ist nach dem Urteil des Musikwissenschaftlers Edgar Istel dementsprechend die ,Anschaulichkeit` der verwendeten Sprache. Er formuliert: „Anschaulichkeit ist die erste und wichtigste Forderung an den musikalischen Dramatiker. Fast alle anderen Gesetze der dramatischen Dichtung lassen sich aus dieser Grundforderung herleiten." (Istel 1914, 75) Vom Librettisten wünscht Istel sich: „Er schaffe anschaulich, d. h. er denke in jedem Augenblick daran, daß das, was er gibt, nicht nur gehört, sondern auch gesehen werden soll." (Istel 1914, 72; Hervorh. im Original) Diese Forderung nach Anschaulichkeit lässt sich nach Overbeck mit der von ihr quantitativ verifizierten Tatsache untermauern, dass bei der gebotenen Kürze eines Librettos die „inhaltsreichste[n] Wortarten“ (Overbeck 2011, 148), in erster Linie die Substantive (dann auch Adjektive und Verben) mit Schlag-oder Schlüsselwortcharakter dominieren (cf. ebd., 229f. und 336). Die Abschnitte zur Isotopie im ersten Teil des Artikels (cf. Agnetta 2017, 9ff.) stützen diesen Befund. Nach Istel soll aber nicht nur der Librettist der Forderung nach Anschaulichkeit entsprechen, sondern es müsse auch der Komponist „mit den Augen, nicht nur den Ohren komponieren“ und auf diese Weise „[n]icht an der Szene vorbeikomponieren, wie das sogar berühmte Komponisten gelegentlich machen" (Istel 1914, 74; Hervorh. im Original). In unterschiedlichen Epochen der Operngeschichte geht das zunächst einmal nicht weiter definierte Ziel, eine ,anschauliche Musik ${ }^{`}$ zu erschaffen, mit jeweils unterschiedlichen Implikationen einher. Welche für italienische Barockopernkomponisten der ersten Hälfte des 18. Jahrhunderts maßgebend sein mussten und welche Rolle dabei das Sprachbild spielt, sei im Folgenden dargelegt. 


\section{Bildhaftigkeit als Brücke zwischen Sprache und Musik}

Nicht nur die Dominanz inhaltsträchtiger Wörter macht einen Librettotext anschaulich, sondern auch der Gebrauch von Metaphern, Vergleichen, Allegorien und Gleichnissen. Die Textgrundlage prototypischer italienischer Barockarien rekurriert, wie im ersten Teil dieses Artikels aufgezeigt wurde, auffällig oft auf eine bildhafte Sprache und offenbart damit eine konzeptuelle Verwandtschaft zur neuzeitlichen Emblematik. Der Librettist gebraucht eine solche aber nicht alleine der Sprachbilder wegen, sondern gerade auch im Hinblick auf die nachträglich stattfindende Vertonung (und Inszenierung ${ }^{1}$ ), denn der Tonsetzer profitiert von dem ihm vorgesetzten Sprachbild, nimmt dieses als Ausgangspunkt für die ,passende 'musikalische Ausgestaltung des Gesangsparts und der Instrumentalstimmen der Arie. Um es mit den Worten eines berühmten Musikkritikers und -theoretikers des 19. Jahrhunderts zu sagen: Die „Malerei des Gleichnisworts“ fungiert dem barocken Komponisten als „Vorwand“ (Marx 1863, 39) für die eigene kreative Arbeit. Nicht selten hat sogar der Komponist dem Dichter die konzeptuelle Metapher vorgegeben, die dieser in Form textueller Metaphern in Verse setzt. Marx’ eigene Metapher von der „Malerei“ des Gleichnisses, das dem Arientypus seinen Namen gibt, stützt die Rede von der Anschaulichkeit bzw. von den bildlichen Inhärenzen des Arientextes. Es ist ebendiese implizit oder explizit geforderte Anschaulichkeit, die auch Carlo Goldoni, welcher die Rezitative des von Apostolo Zeno verfassten GriseldaLibrettos auszubessern und dessen Arien komplett zu ersetzen beauftragt war, dazu bewog, die ursprünglichen, nur punktuell mit konventionellen Metaphern ausgestatteten Verse

\footnotetext{
Vado a mirare un volto, vado a baciare un labbro, per vezzo più gentile, più vago per beltà.

Per te già'l cor disciolto ama in prigion non vile perder la libertà.
}

durch Gualtieros durchgängig die Seemannsallegorie aufgreifende Arie (I.3; s. Agnetta 2017, 5) auszutauschen. So charakterisiert auch Bruni (1971, 270) Zenos Verse als „lindi e contegnosi, che a Vivaldi non avrebbero detto assolutamente niente", weil sie eben jenes eindrucksvollen Imaginariums entbehren, welche die Rede vom Schiffer in Seenot dem Komponisten eine angemessene Vertonungsgrundlage bieten kann.

Letztlich kann die prototypische Gleichnisarie als das Ergebnis eines zweistufigen Übersetzungsprozesses angesehen werden. ${ }^{2}$ Die Darstellung eines in die Dramenhandlung eingebetteten ,eigentlichen' Gefühlszustands bzw. einer passenden, weil unmittelbar auf das Geschehen applizierbaren moralischen Handlungsmaxime wird intralingual anhand eines Sprachbildes wiedergegeben und dieses wiederum motiviert den Einsatz altersemiotisch adäquater Gestaltungsmittel - im Falle der Gleichnisarie eben der Musik. Wichtig ist aber 
anzumerken, dass in diesem Kontext die zwei genannten Übersetzungsprozesse keine $E r$ setzungsprozesse sind, dass also Gleichnis und Musik als intralinguales bzw. intersemiotisches Translat zum jeweiligen Transferendum hinzutreten. In letzter Konsequenz formen sie ein polysemiotisches Ganzes, das durch synsemiotische Bezugnahmen charakterisiert ist. ${ }^{3}$ Die metaphorische Strophe greift, wie gezeigt worden ist (cf. Agnetta 2017, 9ff.), die Isotopieebenen der eigentlich zu übermittelnden Verhaltensmaxime in der zweiten Strophe auf, schafft aber als neu hinzutretender Text auch Raum für neue, konkret-bildhafte Isotopien. Auf diesen kann der vertonende Komponist aufbauen und adäquate musikalische Gestaltungsmittel auswählen, die aus der textlichen eine „synsemiotische Kohärenz“ (cf. Agnetta 2018) zwischen Text und Musik werden lassen. Aus einer semiotischen Perspektive können also die metaphorische Beschreibung in der ersten Arienstrophe und die musikalische Gestaltung in dem Maße als adäquat bezeichnet werden, in dem diese vorgegebene Isotopien wiederaufgreifen. Neben der epistemischen Funktion, den Rezipienten über den Bezug auf einen ihm wohl bekannten Lebensbereich an einen abstrakten Sachverhalt heranzuführen (cf. Agnetta 2017, 8), wirken Metaphern, ferner Metonymien und auch Phraseologismen, als Bindeglieder bzw. Relais zwischen den Elementen der unterschiedlichen Zeichensysteme. ${ }^{4}$ Dieser ,Umweg ${ }^{\prime}$ über die metaphorische Strophe ist gewissermaßen notwendig, standen doch, wie Strohm (2008/I, 102) darlegt, der Komponistengeneration Vivaldis noch nicht sehr viele musikalischen Mittel zum unmittelbaren Gefühlsausdruck zur Verfügung:

A musical metaphoric language directly expressing 'anger', 'grief', 'love', 'hope' etc, could not be fully attained in Vivaldi's time. An allegorical vocabulary of music would have offered the composer long lists of musical 'figures' (a term derived from rhetoric), and perhaps time symbols such as long and short notes, to express visual expressions, motions, natural sounds - but a very short list of figures that could directly express states of mind.

Durch das Aufgreifen und Ausdeuten der textuellen Metaphern bezieht die Musik in der Gleichnisarie ihre Anschaulichkeit also unmittelbar von derjenigen der Sprache. Die folgenden Ausführungen sollen am Beispiel der vier bereits im ersten Teil des vorliegenden Beitrags im Hinblick auf den Text besprochenen Arien (cf. Agnetta 2017, 3 und 5ff.) aufzeigen, inwiefern das vom Librettisten gewählte Sprachbild die Gesangspartie, aber auch die Instrumentalbegleitung derselben bedingt. Wie in den folgenden Ausführungen deutlich wird, ermöglicht nämlich der Rückgriff des Texturhebers auf eine bildreiche Sprache dem Komponisten seinerseits die Gestaltung einer eigenen Tonsprache.

Die Verwendung von Metaphern ist für Libretti der Barockzeit alles andere als unüblich. Innere emotionale Zustände werden durch ebenso eindrucksvolle wie konkrete, meist der $\mathrm{Na}$ tur entnommene Phänomene veranschaulicht. Die vier im vorliegenden Artikel interessierenden Arien „Se ria procella“ (Gualtiero, I.3), „Vede orgogliosa l'ond““ (Ottone, I.5), „Agitata da due venti“ (Costanza, II.2) und „Dopo un'orrida procella“ (Ottone, III.6) vereint allesamt eine Schifffahrtsmetaphorik. Für musikalisch-semantische Untersuchungen von Belang ist 
hier aber v. a. die Beobachtung, dass diese Überführung eines seelischen Zustandes auf eine reale, beschreibbare Situation sowohl in der Sprache als auch in der Musik denotativer Elemente bedarf (oder solcher Elemente, die im Nachhinein als denotativ interpretiert werden). Da Schifffahrt nicht gleich Schifffahrt ist, einmal gefährlich und turbulent (cf. I.3, II.2, III.6), einmal angenehm und ruhig (I.5, III.6) sein kann, kommen ganz unterschiedliche musikalische Mittel zur Ausdeutung des die gleiche Isotopie bedienenden, aber eben einen unterschiedlichen Akzent setzenden Arientexte zum Tragen. Die zum Text hinzutretenden musikalischen Gestaltungsmomente weisen dennoch - wie die folgenden Ausführungen aufzuzeigen bestrebt sind - eine starke, auch nummern- und sogar werkübergreifende Interdependenz auf.

Vertonen italienische Barockopernkomponisten einen Arientext mit einer Meeres- und Schifffahrtsmetaphorik, kann in den meisten Fällen davon ausgegangen werden, dass das Bild der stürmischen See bestimmend ist. Auf diesem Weg wird ein inneres AufgewühltSein der handelnden Figur zum Ausdruck gebracht, der die jeweilige Arie beschieden ist. Dies ist zum Beispiel der Fall in I.3, II.2 und III.6. Die zu diesem Zweck verwendeten Klangmittel sind ein schnell angesetztes Grundtempo (I.3: Allegro; II.2: Allegro; III.6: Allegro molto $)^{5}$, ein sich in der Häufung kleiner Notenwerte manifestierender rasanter Rhythmus der Instrumentalstimmen (I.3, T. 3ff.; II.2, T. 4ff.; III.6, 1ff.) und der Gesangslinie (z. B. I.3, T. 18ff.; II.2, T. 28ff.; III.6, T. 14ff.), schnelle Tonwiederholungen (I.3, T. 5f.; II.2, T. 4ff. und 28ff.; III.6, T. 8ff.), stets auf unterschiedlichen Tonhöhen auszuführende Melodiefragmente (I.3, T. 15f.; II.2, T. 54ff.; III.6, T. 20f,), wellenförmig auf- und absteigende Dreiklangsbrechungen (I.3, II.2, III.6, jeweils bereits ab T. 1ff.) und Tonleitern (I.3, T. 18ff.; II.2, T. 51ff.; III.6, T. 14ff.), synkopierte, d. h. dem Grundschlag des Takts zuwiderlaufende Rhythmen (I.3, T. 5ff.; II.2, T. 48f.) sowie mehr oder weniger große, für den Gesangssolisten oft unsangliche Intervallsprünge (I.3, T. 13f.; I.5, T. 11f. und 17f.; II.2, T. 24ff.; III.6, T. 8f.). Dabei ist es nach Strohm eine u. a. genuin von Vivaldi eingeführte Neuerung gewesen, dass die ausdeutenden Momente nicht nur - und auch nicht primär - in der Gesangsstimme, sondern nun auch in den Instrumentalritornellen zu suchen sind: „Dabei übernahm die musikalische Vertonung einen immer größeren Anteil der dramatischen Aufgabe; es handelte sich um eine ,Dramatisierung' der Musik ebenso wie um eine ,Musikalisierung" des Dramas" (Strohm 1995, 1487; cf. auch Strohm 2008/I, 105).

Die Mimesis des in der ersten metaphorischen Arienstrophe skizzierten Szenarios durch die Musik erschöpft sich nicht nur in der Lautmalerei, d. h. in der Imitation akustischer Ereignisse (Wind und Donner), sondern manifestiert sich auch in der musikalischen Nachzeichnung optisch wahrnehmbarer Phänomene (Wellen- und Schiffsbewegung). Und nicht nur die Instrumentalstimmen zeichnen das in der metaphorischen Strophe beschriebene Naturereignis nach, auch der hör- und wohl auch sichtbare Aufwand in der Produktion der Koloraturen durch den Gesangsinterpreten wird zum Zeichen für die Gefahren einer stürmischen See. Exemplarisch kann dies etwa an Ottones Arie „Dopo un'orrida procella“ betrachtet werden, in welcher sein Imponiergehabe in virtuosesten Koloraturen kulminiert und in welcher der explizit genannte und reich verzierte Schlüsselbegriff „affanno“, der Liebesbemühungen und Unwetterangst gleichsetzt, auch für den Sänger zum Kraftakt wird: 


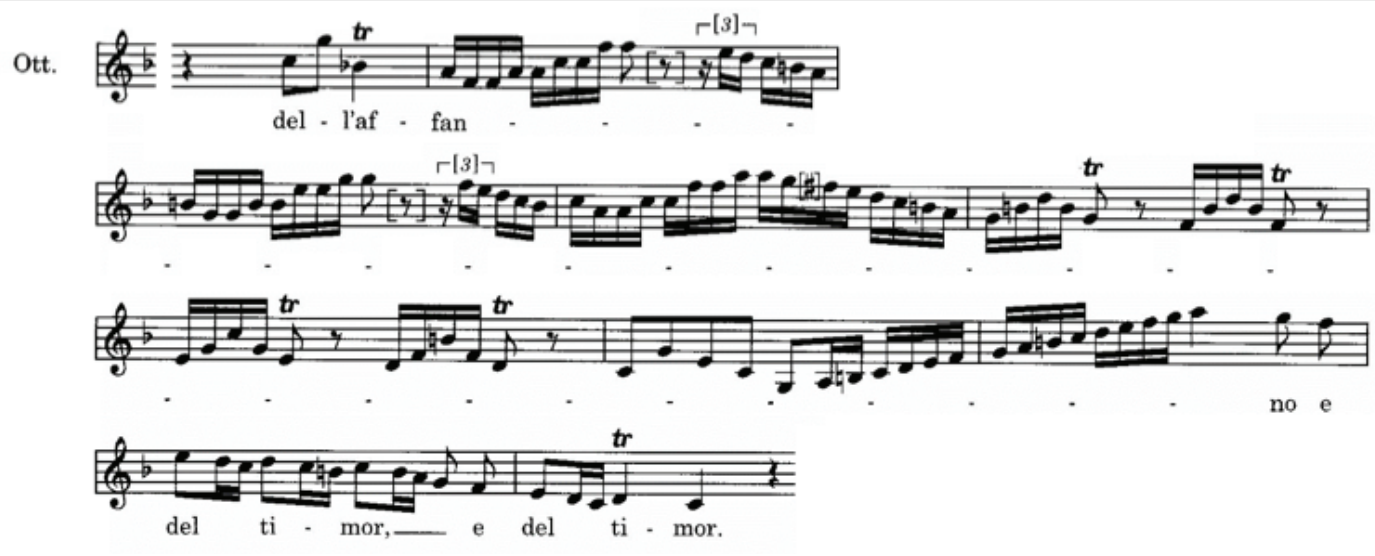

Notenbeispiel 1: Ottone, „Dopo un'orrida procella” (Griselda III.6, T. 16ff.)

Ergänzt werden solche und andere Arienstellen, wie Strohm (2008/I, 103) weiterhin ausführt, in den meisten Fällen durch die Gestik und Mimik des Gesangsinterpreten, durch weitere semiotische Ressourcen also, auf welche die Aufführung der Oper basiert. Auch diese neuen Zeichen müssen ebenso zur im sprachlichen wie musikalischen Text eingeschriebenen Gesamtaussage passen und Isotopien wiederaufgreifen:

[I]n fact, Vivaldi's imitative strategies cannot be completely understood without considering the stage performance: the actor's choice may sometimes have been to complement rather than duplicate the musical imagery. Storm music, for example, might be performed in a statuesque fashion but with expressive gesture and mime (wringing hands, sorrowful facial expression) to represent the character's inner emotions. However, gesture, mime, verbal rhetoric and musical imagery probably coincided often enough.

Aus dem pragmatischen Blickwinkel betrachtet, entsprechen Ausdeutungen wie die im Notenbeispiel 1 nicht nur der durchaus intrinsischen Motivation des Komponisten, einen adäquaten Emotionsausdruck auf der Bühne zu ermöglichen, sondern befriedigt auch die extrinsisch zu bezeichnende Lust der ausführenden Sänger und des Publikums an der Produktion respektive Rezeption virtuoser, reich kolorierter und letztlich auch effekthaschender Gesangseinlagen.

\section{Zeichenlernprozesse bei der Arienrezeption}

Oft wird von Seiten einer die Sprache und die Musik kontrastierenden Semiotik in verkürzender Weise allein bestimmten verbalen Zeichen die Stellung als Autosemantika zugestanden, weil diese in der Regel auf der langue-Ebene einen definierten Bedeutungsumfang aufweisen. Der 
Musik wird dagegen pauschal semantische Vagheit, wenn nicht gar Asemantizität attestiert (cf. z. B. Kneif 1990, 135; Harweg 1994, 147), weil sie vergleichbar eindeutige SignifikantSignifikat-Verknüpfungen auf der Systemebene oft entbehrt. ${ }^{6}$ Von einer solchen Anschauung zeugt auch eine Aussage Strohms zu Vivaldis Arien in der Griselda-Oper, wenn er schreibt: „Darstellenden Charakter hat vor allem seine [i.e. Vivaldis] Instrumentalbehandlung, doch ist sie unspezifisch - die Unterschiede zwischen den verschiedenen Gleichnisarien verschwimmen." (Strohm 1979, 233) Bestimmte musikalische Mittel würden von Vivaldi zur Ausdeutung ganz heterogener Texte herangezogen werden, seien deswegen unpassend und wenig glaubhaft; jedenfalls könne, führt man Strohms Gedanken zu Ende, hier nicht von musikalischen Zeichen gesprochen werden. Im vorliegenden Artikel soll ein anderer Blickwinkel bestimmend sein: Hier interessiert nicht primär die Fragestellung, ob sich die Musik gewissermaßen auf der langue-Ebene als Zeichensystem beschreiben und sich jeder existierenden musikalischen Figur eine klar umrissene Bedeutung zuweisen lässt. Es gilt vielmehr die Inhaltsseite der faktisch in einer Arie Verwendung findenden musikalischen Gestaltungsmomente zu beschreiben - unter anderem auch auf ihr Zusammenspiel mit dem ebenso faktisch vorliegenden Text hin. Ziel ist also die (Re-)Konstruktion einer zunächst arien-, dann auch werkinternen Semantik, nicht der Umriss einer werkunabhängigen, allgemeingültigen und statischen ,Semantik der Musik'. Wie bei rein sprachlichen Artefakten von der Semantik auf der parole-Ebene, gewonnen etwa durch texthermeneutische Methoden, die Rede sein würde, ${ }^{7}$ so lässt sich auch die Inhaltsseite einer zum Zeichen werdenden musikalischen Figur erst in Auseinandersetzung mit einem konkreten Musikstück schrittweise konturieren. ${ }^{8}$

Aus der Sicht der Polysemiotizitätsforschung geht es um die konkrete Interaktionsweise heterosemiotischer Elemente, auch wenn diese Interaktion mit den Grenzen des Werks enden und nie eine gesamtgesellschaftliche Konventionalisierung erfahren, die für manchen Semiotiker Definitionsmerkmal des Zeichenhaften darstellt (etwa für Fricke 2000, 103f.). Wie oft richtig hervorgehoben, entbehren musikalischen Strukturen womöglich eine genau umrissene und feststehende Bedeutung. Sie werden von einem Komponisten und später von seinen Rezipienten und Kritikern eher mit einem werkspezifischen Sinn in Verbindung gebracht. Dieser Sinn und letztlich auch die Einschätzung, ob die konkreten musikalischen Mittel zu dessen Konstitution geeignet erscheinen, sind in vielen Fällen eine Angelegenheit subjektiver Natur. Dies vorausgeschickt, muss aber auch betont werden, dass es auch in der Musik bestimmte Topoi gibt, die dem damaligen und auch heutigen Publikum wohl geläufig sind und diesem als Interpretationsschemata zur Verfügung stehen. Die häufig zu beobachtende Stereotypie von Aufbau, Gehalt und musikalischer Gestaltung einer barocken Gleichnisarie etwa begünstigt in besonderer Weise ihre rasche Kontextualisierung, Erschließung und letztlich ihr Verständnis (s. u.). Nach Bruni nimmt die Konventionalität der Machart bis ins 18. Jahrhundert hinein den gleichen Stellenwert ein, den in der Opernbranche des 20. Jahrhunderts und von heute die Repertoirebildung und -pflege innehat: „Sul piano stilistico-musicale, un surrogato, in un certo senso, del concetto di repertorio può essere identificato nell'uso ricorrente, da parte dei compositori, di formule 
e stampi fissi che il pubblico riconosceva all'ascolto e collegava ogni volta a un'esperienza emotiva già nota." (Bruni 1971, 268) Auch die oben genannten Motivbausteine, die gleich in mehreren Gleichnisarien vorkommen, dürften wohl als solche musikalischen Zeichen gelten, v. a. die wellenartigen Koloraturen in der Arie Gualtieros (I.3, T. 21f.) sowie Constanzas (II.2, T. 51f.), die Arpeggien in Ottones erster (I.5, s. u.) und der Einsatz der Hörner in seiner zweiten Seemannsarie (III.6, T. 1ff.). Solche musikalischen Figurationen und Instrumentierungen sind als konventionelle Zeichen zu fassen, weil sie sich in zeitgenössischen Abhandlungen zur musikalischen Rhetorik sowie in den Rezensionen und Werkkritiken genau kodiert finden.

Weil die Musik gemeinhin als semantisch vage begriffen wird, bedarf sie nach dem Zeugnis bestimmter Theoretiker immer heterosemiotischer Autosemantika, etwa eines Textes oder eines Bildes als einer monosemierenden, verständlich machenden Entität. „Die Tonkunst“, so schreibt beispielsweise Heinrich Christoph Koch, kann „eigentlich nur in Verbindung mit der Dichtkunst ihre höchste Absicht, ihren eigentlichen Endzweck erreichen“, weil sie der Vereindeutigung durch poetische „Begriffe und Bilder“ (Koch 2007, 223) bedarf.9 Man beachte diese letzte Ergänzung, werden doch hiermit die ,Bilder der Poesie', d. h. auch die Metaphern, explizit als monosemierende Größen genannt. So kommt es häufig vor, dass Sinn und Bedeutung musikalischer Strukturen „erst einmal auf irgendeinem Umweg festgestellt werden, ehe es zum Bewußtsein und Durchfühlen der symbolischen Zusammenhänge kommen kann, die der Komponist im einen oder andern Sinne beabsichtigt hat" (Schering 1990: 44). Solche „Umwege“ bestehen werkintern durch die Verbindung der Musik mit Text und Inszenierung und werkextern beispielsweise in der Rezeption von Werkeinführungen, Plakaten etc. sowie dem Studium musikwissenschaftlicher Abhandlungen. Die häufig zu beobachtende Notwenigkeit einer Semantisierung der Musik über solche Umwege bedeutet nicht, dass sich musikalische Zeichen nicht auch von dieser ihrer monosemierenden und verständlich machenden Grundlage emanzipieren können, wie die folgenden Ausführungen zeigen wollen. ${ }^{10}$

Wie in ähnlichem Kontext gezeigt (vgl. Agnetta 2018), kann in kombinierten, d. h. polysemiotischen Kommunikaten a priori von der Musik als semiotischer, und das bedeutet nicht allein auf syntaktischer und pragmatischer Ebene beschreibbarer, sondern auch an der Sinnkonstitution des Werkes beteiligter Größe ausgegangen werden; denn der Komponist wählt musikalische Mittel aus, die zum Text passen und zu den globalen Aussagen des fertigen Werkes führen. Die der Musik in den Philologien und selbst der Musikwissenschaft oft attestierte Vagheit erklärt sich u. a. durch ihre Begriffslosigkeit und besonders durch ihre Unfähigkeit, Abstrakta wie ,Liebe', ,Schönheit' genau zu benennen (cf. Cook 2007, 100f.). Musik ist dennoch in der Lage, an der Emergenz von Bedeutung beizutragen, indem sie konkrete „energetische[...] und expressive[...] Eigenschaften“ (ebd., 101) verkörpert, die einen Rezipienten zur Deutung bzw. Umdeutung ihres Ko- oder Kontextes veranlassen. Ich nenne diese Eigenschaften bzw. Merkmale in Anlehnung an die (sprach-) semiotische Forschung, Seme' und deren Rekurrenz in allen beteiligten Ausdrucksformen (Musik, Sprache, Szene) bezeichne ich als ,Isotopie‘. Cook spricht im ersten Fall von 
„Nuancen“ (ebd., 100f.), im zweiten von „Verschmelzungsraum“ (ebd., 104). Unabhängig von der gewählten Terminologie ist die Beobachtung zu unterstreichen, dass die Auswahl der Seme bzw. Eigenschaften der in einem kombinierten Kommunikat interagierenden Zeichen von Rezipienten vorgenommen wird, die einen jeweils unterschiedlichen Sinn konstruieren, je nachdem ob sie die Potenziale und Konventionen musikalisch intendierter Bedeutungszuweisung kennen oder nicht.

Die Musik der Gleichnisarie ist losgelöst von ihrem musikdramatischen Kontext in den meisten Fällen nicht die Trägerin einer einzigen, wohldefinierten Bedeutung und gewinnt also häufig erst mit dem Vortrag der Arienverse und in der Einbettung in eine Szene genauere semantische Konturen. Text und Inszenierung wirken monosemierend. Um das Verhältnis von Musik und Librettotext näher zu betrachten, stellt es sich für die Semiotik wie so oft als besonders reizvoll heraus, Begriffe aus der Sprachwissenschaft heranzuziehen und deren Nutzen und Adäquatheit für die Beschreibung geistesverwandter Phänomene in anderen Zeichensystemen sowie des Zusammenspiels unterschiedlicher Zeichen kritisch zu diskutieren. In diesem Fall handelt es sich um Termini, die Beaugrande/Dressler geprägt haben, nämlich um den der „Koreferenz" zwischen einem Autosemantikum und seinen sogenannten „Pro-Formen“. Letztgenannte werden beschrieben als

ökonomische, kurze Wörter ohne besonderen Inhalt, die für determinierte, inhaltsaktivierende Ausdrücke an der Oberfläche des Textes einstehen können [...]. Diese Pro-Formen erlauben es Textbenutzern, den Inhalt im aktiven Gedächtnisspeicher handhabbar zu halten [...], ohne alles wiederholen zu müssen. Die bekanntesten Pro-Formen sind die Pronomina, die die Funktion von Hauptwörtern oder Nominalphrasen erfüllen, mit denen sie koreferieren (d. h. Referenz [...] gemeinsam haben). (Beaugrande/Dressler 1981, 64; Hervorh. im Original)

Greift man den oben erwähnten Topos auf und sieht in kombinierten Zeichenkomplexen wie den Arien die vielen Inhaltswörter im Text als Autosemantika und die Musik als hinzutretendes und, irgendwie' am Zeichengeschehen beteiligtes Element an, so kann auch hinsichtlich dieser Beziehung von einer „Koreferenz" die Rede sein. Die Musik ,koreferiert" mit dem Librettotext und hier vornehmlich mit der metaphorischen Textstrophe einer Arie, verweist mit ihr auf dieselbe zu imaginierende Situation. Wie bei den sprachlichen ProFormen ist ihr Referenzobjekt zunächst kontextabhängig, d. h. abhängig von den mit ihr koreferierenden Autosemantika im jeweiligen parole-Akt. Auf der langue-Ebene hat sie kein konkretes Referenzobjekt.

Und doch unterscheiden sich die musikalischen Mittel in der Arie auch von den durch Beaugrande/Dressler beschriebenen Pro-Formen. Ihr Einsatz entspricht im Vergleich zu diesen nicht unbedingt einem Willen zur weitgehenden kommunikativen Ökonomie, denn zumal in den Gleichnisarien ist die Produktion einer textlichen Aussage in und mit Musik in vielerlei Hinsicht aufwendiger als ohne sie. Auch fungieren die musikalischen Elemente nicht einfach als Platzhalter für die Autosemantika des Arientextes, sondern sind 
stets mehr: Sie ergänzen, modifizieren und verneinen sogar manchmal die textliche Aussage. Die Sinndimensionen einer Gleichnisarie sind nicht die gleichen, wenn man allein ihre sprachliche Grundlage, den Arientext, interpretiert. Gerade dies besagt ja die Beschreibung eines polysemiotischen Komplexes als übersummative Gestalt (cf. Agnetta 2017, 4): Das Ganze ist mehr als seine Teile, die an der Oper erfahrbaren Sinndimensionen sind andere als die am Libretto beobachtbaren. Von einer solchen Ansicht entfernt sich allerdings ein Urteil Albert Giers (1999: 6), der schreibt:

\begin{abstract}
Nehmen wir etwa die in der Barockoper so beliebten Gleichnisarien. Der Text schafft hier einen Anlaß zu illustrativer Vertonung: Wenn der Protagonist seine seelische Erschütterung mit einem Sturm auf offenem Meer vergleicht, schlagen im Orchester die Wellen hoch; ist im Text von der Geschicklichkeit des Jägers die Rede, gibt das Gelegenheit zu einem Hornsolo, und so weiter. Dabei fügt die Musik nichts hinzu, was nicht schon im Text enthalten wäre, sondern verdoppelt dessen Aussage, die Arie als Einheit von Wort und Ton ist somit übercodiert.
\end{abstract}

Die Interdependenz von Sprache und Musik in der Gleichnisarie erschöpft sich m. E. nicht in der intensivierenden, nach Gier letztlich wohl meist als redundant zu bezeichnenden Funktion der Letztgenannten. Dass dem nicht immer so ist, kann anhand Ottones Arie "Vede orgogliosa l'onda“ (III.6) veranschaulicht werden, in der die Textaussage durch die Musik modifiziert, in diesem Fall vereindeutigt wird: Rein textlich gesehen hätte die Arie in der gleichen Weise vertont werden können wie die übrigen Schifffahrtsarien, nämlich unter Rückgriff auf ein rasches Tempo, reichlich bewegte Koloraturen, weite Intervallsprünge, etc. (s. o.). Dies wäre durchaus legitim gewesen, beinhaltet doch der Arientext die vom Komponisten hierzu benötigten Formulierungen („orgogliosa l'onda“, „mare infido“, „nocchiero ardito“, „adonta del rigore“). Doch nimmt diese Nummer im Kontext der eben beschriebenen Arien eine Sonderstellung ein: In ihr wird mit den musikalischen Gestaltungsmitteln primär das Bild eines zuversichtlichen, nicht von den Gefahren der stürmischen See heimgesuchten Seemannes vermittelt. Vivaldi liefert durch eine, aus der Reihe fallende' Vertonung also eine bestimmte Interpretation des Arientextes. Gewählt wurde ein langsames Grundtempo (Largo), eine G-Dur-Harmonik sowie eine mit dem 4/4-Metrum weitgehend konformgehende Melodik. Ondulierende Arpeggien in den hohen Streichern simulieren eine konstante Bewegung und vermitteln gemeinsam mit den Tonwiederholungen in der melodietragenden Stimme sowie in den begleitenden Bassstimmen eine gewisse Ruhe. Die langsam auszuführenden großen Intervallsprünge (T. 12 und 17ff.), Melismen (T. 30f.) und die fortepiani (T. 19f., 34f.) sind hier nur eine relativ schwache Erinnerung an die Bedrohungen der stürmisch bewegten Meereswellen. Bestimmend ist zudem die sehnsuchtsvolle Aufwärtsrichtung der Motive auf der Formulierung „spera di ribaciar" (T. 17f., 31-33). 
Ott.

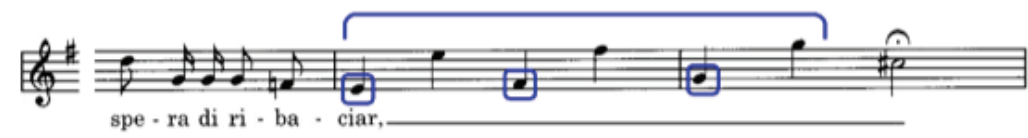

Ott.

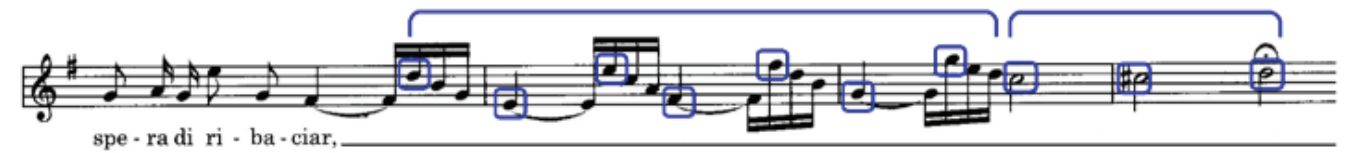

Notenbeispiel 2: Ottone, „Vede orgogliosa l'onda” (Griselda I.5, T. 16f. und 31-33)

Die Zukunftsgerichtetheit der Verbalphrase ,spera di ribaciar', das momentane Ausbleiben eines sehnlichst erwünschten Zustands, wird durch die Aufwärtsrichtung der Tonsequenz $\left(e^{\prime}-f^{\prime}-g^{\prime}\right)$, deren Doppelung in einer höheren Oktavlage sowie durch die Fermate auf der zunächst unaufgelöst bleibenden Doppeldominantharmonie (A-Dur, mit Leitton in der Gesangsstimme) wiederaufgegriffen.

Strohm (1979, 229f.) äußert sich zur dramatischen Glaubwürdigkeit dieser entlehnten und auch in Vivaldis Oper Adelaide (ebenfalls 1735) wiederverwendeten (vgl. Strohm 2008/ II, 573) - Arie folgendermaßen:

Ottones „Vede orgogliosa l’onda“ zum Beispiel hatte ursprünglich einen äußerlich ähnlichen Text [...], der aber die Verzagtheit eines Wanderers in der Nacht zum Symbol nimmt, nicht die Zuversicht des Seemanns wie im zweiten Text. Vivaldi hat die Musik nicht geändert, sondern nur den neuen Text unterlegt.

Er bezeichnet die Arie dementsprechend als „rüde Kontrafaktur“ (Strohm 1979, 233). Aus diesem Zeugnis spricht wiederum die Ambition, musikalischen Strukturen kontextunabhängige Bedeutungen zuweisen zu wollen, statt den konkreten Interdependenzen und der Adäquatheit der vorliegenden Vertonung mit den unterschiedlichen Texten, der genannten Nachtwanderung zum einen und der ruhigen Seefahrt zum anderen, nachzuspüren. Dass im Übrigen die musikalischen Mittel, etwa die begleitenden Arpeggien, besser zum Nachtwanderer denn zum Schiffer passen (eine These, welche die Rede von der „rüden Kontrafaktur“ überhaupt legitimieren würde), sei dahingestellt, findet diese Begleitfigur doch beispielsweise bereits im die Schifffahrtsmetapher ebenso aufgreifenden Accompagnato-Rezitativ von Bachs Kreuzstabkantate (BWV 56) einen heute berühmten Vorgänger, wenn sie auch anders als „Vede orgogliosa l’onda“ - der Textaussage („Mein Wandel auf der Welt / ist einer Schifffahrt gleich: / Betrübnis, Kreuz und Not / Sind Wellen, welche mich bedecken [...]") des deutschen Rezitativs entsprechend - vom ursprünglichen B-Dur schnell zu einer dominantischen und Moll-Harmonik (ab T. 3) wechselt: 


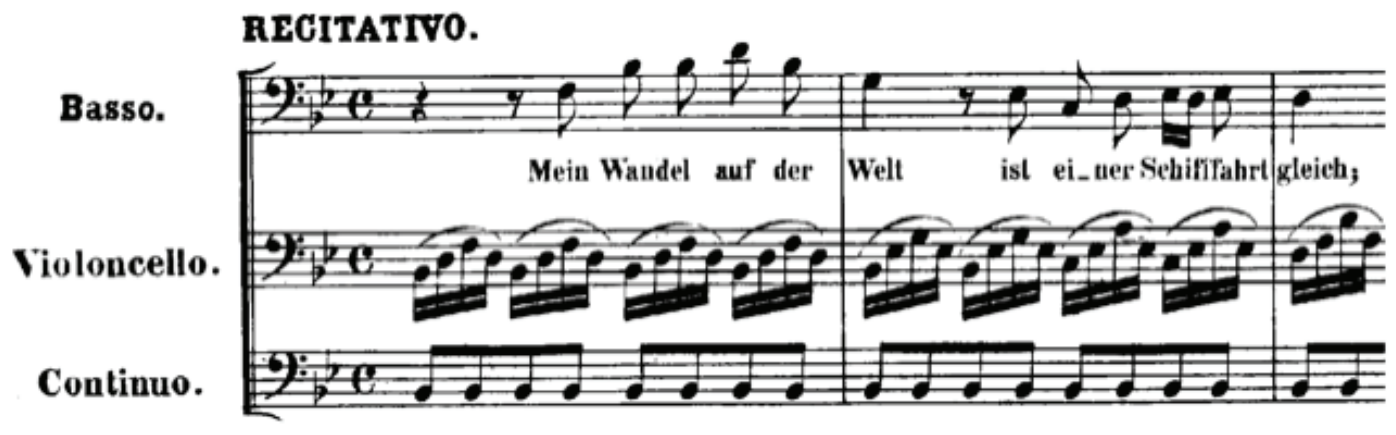

Notenbeispiel 3: „Mein Wandel auf der Welt“ (J. S. Bach, BWV 56, Recitativo, T. 1ff.)

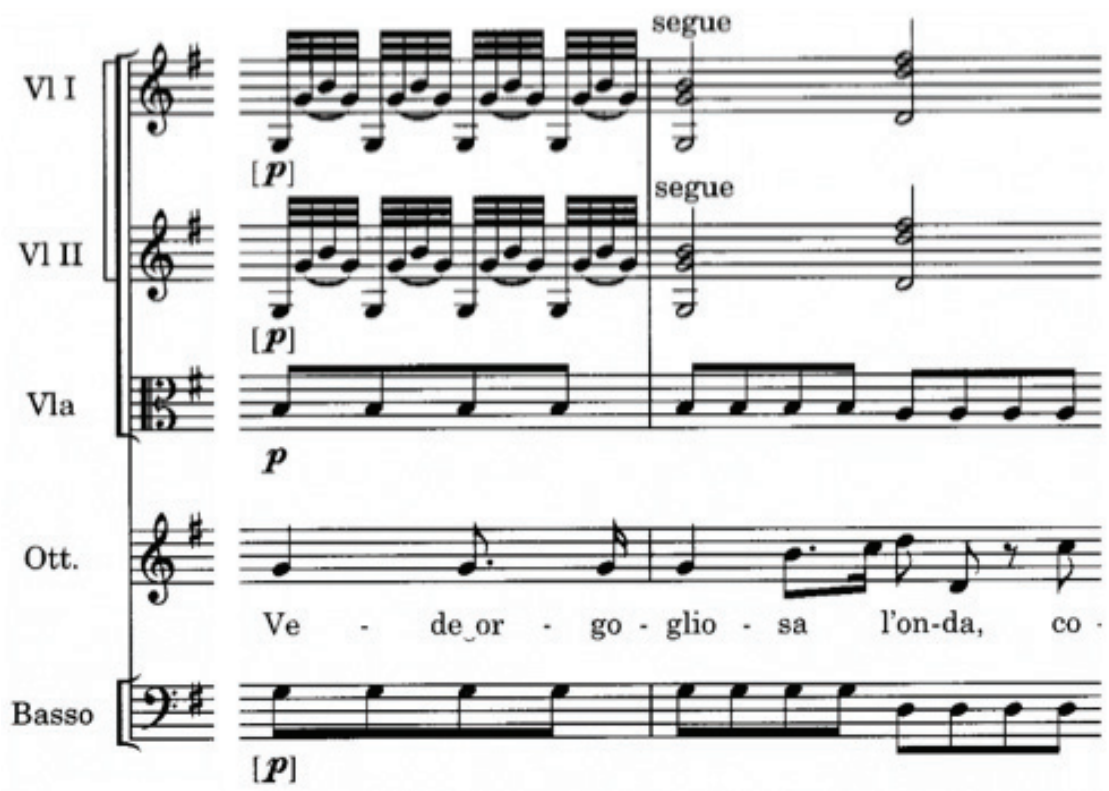

Notenbeispiel 4: Ottone, „Vede orgogliosa l'onda” (Griselda I.5, T. 10f.)

In jedem Fall ist der Interpret bei der Rezeption der Arie dazu angehalten, Musik und Arientext aufeinander zu beziehen. Dabei kommt es vor, dass die musikalischen Strukturen semantisch aufgeladen werden, Zeichenlernprozesse werden initiiert. Am Aufbau prototypischer Gleichnisarien lässt sich das werkinterne Zeichenlernen gut veranschaulichen: Die Form der Gleichnisarie, meistens eine Da-capo-Arie, greift die strophische Zweiteilung des Textes auf. Auf ein rein instrumentales Vorspiel folgt die meist zweifach in ihrer Gänze wiederholte erste, metaphorische Strophe. Ein Zwischenspiel leitet zur zweiten, textlich wie musikalisch zum bereits Gehörten in Kontrast stehenden, nichtmetaphorischen Strophe über, nach welcher der gesamte A-Teil (Vorspiel, erste Strophe mit Wiederholungen und Nachspiel) noch einmal aufgegriffen wird. Der im B-Teil, d. h. in der nichtmetaphorischen 
Strophe herbeigerufene Stimmungs- oder Charakterwechsel, der der inhaltlichen wie musikalischen Kontrastierung dient, ist das arieninterne Signal für die Applikation des bisher metaphorisch Beschriebenen auf die dramatische Situation. Der mehrgliedrige Aufbau der italienischen barocken Gleichnisarie ist damit gekennzeichnet von einer Bewegung vom Intuitiven und Vagen zum Konkret-Bildhaftem, zum Abstrakten und wieder zurück zum Konkreten, wobei die bereits semantisierte Musik im Verlauf der Arie eine veränderte semiotische Qualität erlangt.

Entgegen den bekannten Regeln effizienten Lernens, folgt das Zeichenlernen in der Gleichnisarie nicht der pädagogischen Empfehlung einer Bewegungsrichtung vom Bekannten bzw. Eindeutigen (dem Arientext) zum Unbekannten oder Uneindeutigen (der Musik), sondern es wird das Unbekannte bzw. intuitiv Aufzufassende antizipiert (die Musik), schrittweise expliziert (durch die Metapher) und letztlich auf das Musikdrama, deren Teil die Gleichnisarie ist, bezogen (durch die nichtmetaphorische Strophe). Greift man das oben erwähnte Bild der Arienproduktion als zweistufigen Übersetzungsprozess wieder auf, bedeutet das in diesem Kontext, dass die Zieltexte bzw. Translate (die Musik und metaphorische Arienstrophe) noch vor dem Ausgangstext bzw. Transferendum (die nicht-metaphorische Strophe) rezipiert werden.

Da Autosemantika und die mit ihnen koreferierenden Elemente in Texten nicht zusammen auftreten können, unterscheiden Beaugrande/Dressler die Anaphora, d. h. den „Gebrauch einer Pro-Form nach dem koreferenten Ausdruck“, von der Kataphora, d. h. dem „Gebrauch der Pro-Form vor dem koreferenten Ausdruck“ (1981, 65). Bei einer prototypischen Gleichnisarie tritt nun der Fall auf, den Beaugrande/Dressler für den sprachlichen Bereich als „kataphorische“ Bezugnahme bezeichnet und mit Blick auf den Leseprozess folgendermaßen beschrieben haben: „Zunächst hat er [i.e. der Leser] nur ein problematisches Wissen, da eine Anlehnung an das Wissen des Lesers schwierig erscheint. Die Kataphora fügt ein momentanes Problem in die Oberfläche des Textes ein und spornt den Leser an, in die Geschichte weiter einzusteigen." (ebd., 66; Hervorh. im Original) Bei der Arie verhält es sich ähnlich: Der wohlwollende Rezipient vernimmt das musikalische Vorspiel, das in der Regel dem Inhalt der erst später exponierten verbalen Aussage entspricht. Auch hier ereignet sich für denjenigen, der die musikalische Ausgestaltung verstehen will und sie nicht nur als wohlklingende Unterhaltung begreift, zunächst ein kommunikatives Problem, das aber gleichzeitig sein Interesse bzw. seinen hermeneutischen Ehrgeiz weckt. Das Instrumentalvorspiel der barocken Opernarie übernimmt beim erstmaligen Hören die Funktion einer kataphorisch eingesetzten Pro-Form, die schon vor dem Einsatz des Arientextes die allgemeine Stimmung prägt und damit einen ersten, intuitiven Interpretationsschlüssel für dessen Verständnis liefert. Es ist dabei zu bedenken, dass das werkinterne Zeichenlernen auch durch die Inszenierung, aber auch durch Zusatzmedien wie dem damals meist erschwinglichen Leselibretto wesentlich erleichtert wurde, versetzte das letztgenannte doch den eifrigen Rezipienten in die Lage, den Text einer Arie während oder sogar noch vor dem eigentlichen Erklingen des Arienvorspiels zu rezipieren. 
Was sich der Rezipient bei der ersten Inbezugsetzung von Musik und Text erarbeiten, was er erlernen musste, kann er bereits beim erneuten Erklingen von Text und Musik als mehr oder weniger stabilen Bewusstseinsinhalt abrufen. Die Komponenten der während und nach dem erstmaligen Textvortrag wieder aufgegriffenen musikalischen Motivik in den Zwischen- und Nachspielen (deswegen auch als Ritornelle bezeichnet) können somit als anaphorische Bezugnahmen und dann - wieso nicht? - irgendwann als (nicht nur arienund werkinterne) Zeichen gewertet werden. Die Ergebnisse der durch ein Werk initiierten Zeichenlernprozesse können dem Rezipienten sogar bei einer späteren Wiederaufnahme des Dialogs mit dem gleichen oder einem anderen Kunstwerk von neuem Nutzen sein. Nach und nach werden vom Individuum (in einem bestimmten Rahmen aber auch von einem Kollektiv) Wissensbestände aufgebaut, die mit jeder Rezeption der gleichen Zeichenstruktur bzw. ähnlicher Zeichenstrukturen reaktiviert werden. Eine musikalische Struktur, der vor der Werkrezeption auf der Systemebene kein Zeichencharakter zugesprochen werden konnte, die aber in einer Rezeptionssituation Zeichencharakter erlangt hat, kann bei der erneuten Rezeption bereits als Zeichen gelten. Dies gilt als gesichertes Ergebnis in der Musikpsychologie und -physiologie, die sich mit den Bedingungen musikalischer Semiose beschäftigen: „,[B]ei wiederholter Verarbeitung ähnlicher Reize ändert das Gehirn allmählich seine Antworten, es lernt." (Hesse 1995: 1105) Eine Gleichnisarie dieses Typs verkörpert nicht zuletzt auf diese Weise den Mechanismus des Zeichenlernens, auf den jeder hermeneutische Zirkel aufbaut: Mit jeder Rezeption derselben (oder einer ähnlichen) Struktur, derselben (oder einer ähnlichen) Zeichenkonfiguration kann der Rezipient aus einem immer größeren Wissensbestand - und das bedeutet auch: aus einem immer größeren Zeicheninventar - schöpfen. Insofern ist jede Rezeption und jede erneute Rezeption eine aufeinander aufbauende Folge von „Aneignungsaktivitäten“ (Bucher 2004, 136). Auch dies haben übrigens Emblem, Gleichnis (in Prosa oder Versform) und allegorische Arie gemeinsam: Sie sind - wie man heute sagen würde - didaktisch durchdacht. Die oft wiederholten textlichen und musikalischen Bausteine der Gleichnisarie dienen also nicht nur, wie bereits angemerkt, einer reich ausgeschmückten, virtuosen Gesangslinie als Vorwand, sondern können durchaus einen nicht zu unterschätzenden Stellenwert innerhalb jener über die Operngeschichte hinweg zu beobachtenden Entwicklung innehaben, die manchmal als zunehmende Semantisierung der Musik angesehen wird (Schering 1990, 37).

Im Falle von Vivaldis Griselda kann auf der Werk-Ebene auch die Häufung der Schifffahrtsarien solche Lerneffekte begünstigen. Die Meeresmetaphorik wirkt hier, durchaus im dramatischen Sinne, als „unifying metaphor" (Hill 1978, 67). Ottones Arie „Dopo un'orrida procella" (III.6), in der zwar von einem Unwetter, nicht aber explizit von einem solchen auf See die Rede ist, wird in dieser Hinsicht wohl von vielen Rezipienten als Fortsetzung der werkintern so prominenten Reihe der Seemannsallegorien interpretiert. Die Anwendung dieses Bildes auf ähnliche Gefühlslagen und konkret die Wiederholung von bestimmten Lexemen (z. B. „procella“ in I.3 und III.6) und musikalischen Motiven (etwa das hoheWellen-Motiv) begünstigen diese arienübergreifende und werkinterne Interpretationslinie. 
Trotz der geschlossenen Form der Arie tragen solche Elemente zur Kohärenz des gesamten Werks bei. Die Interpretationsarbeit endet also nicht mit den Grenzen der Arie. Ambitionen zur dramatisch kohärenten Gestaltung der Oper können in der Griselda, die gemeinhin als Pasticcio-Oper gilt (cf. Strohm 2008/II, 585), also durchaus auch beobachtet werden.

\section{Fazit}

Im vorliegenden Beitrag wurde aufgezeigt, wie der Librettist durch stilistische Entscheidungen sowohl auf der Form- als auch auf der Inhaltsebene den Grundstein für das synsemiotische Zusammenspiel von Sprache und Musik in der Oper legt. Zunutze macht er sich die musikalischen und bildlich-szenischen Inhärenzen der Sprache. Betrachtet wurde in diesem Beitrag die Gleichnisarie als der bei italienischen Barockopernproduzenten des frühen 18. Jahrhunderts am meisten verbreitete Arientypus.

Die Musik kann sehr leicht semantisiert werden. Eine Möglichkeit ist die Semantisierung durch Wörter und ein durch sie vermitteltes Bild oder - semiotisch gesprochen - durch Verbalia und deren bildlichen Inhärenzen. Am augenfälligsten sind diese piktorialen oder szenischen Inhärenzen bei bildhafter Sprache, also bei Metaphern, Vergleichen, u. Ä. m. Nachdem im ersten Teil des vorliegenden Beitrags (Agnetta 2017) den Charakteristika und Funktionen der bildhaften Sprache in den Arientexten nachgegangen worden ist, sollte im zweiten Teil an einigen aussagekräftigen Beispielen die Interrelation zwischen den vom Librettisten verarbeiteten textuellen Metaphern und der hinzutretenden Musik in den Vordergrund gerückt werden. Der Fokus ist auf die faktischen Bezugnahmen von Sprache und Musik gelegt worden, die synsemiotisch zum Aufbau von Werksinn beitragen und zu diesem Zwecke auch Zeichenlernprozesse zu initiieren imstande sind.

Die Dynamik des Zeichenbegriffs muss hier explizit unterstrichen werden: Die Verknüpfung zwischen dem musikalischem Signifikanten - sei dieser eine Note, ein Intervall, eine Notengruppe, ein markanter Rhythmus, eine Tonart, ein Wechsel in der Dynamik, eine Instrumentierung, eine Harmonie, ein Harmonieverlauf, die homophone oder polyphone Textur eines gesamten Abschnitts - und dem nahegelegten Signifikat erfolgt arien- und, wie gezeigt worden ist, werkintern. Eine solche Verbindung kann insofern nicht als stabil erachtet werden, als sie keine werkübergreifende Gültigkeit reklamiert und auch nicht als Teil eines stabilen und überzeitlich gegebenen musikalischen Zeichensystems betrachtet werden kann. Die Frage nach der musikalischen Semantik ist also in diesen Fällen primär werkintern zu beantworten. Semantizität darf hier wohlwollend vorausgesetzt werden, denn die faktische Polysemiotizität des musikalischen Dramas macht es erforderlich, dass alle Teile in Hinblick auf einen kohärenten Sinn interpretiert werden. Die Musikalisierung einer Arie steht dabei keineswegs von vornherein fest. Es lassen sich nur wenige und nicht unbedingt verbindliche Konventionen beschreiben, denen der Komponist bei der Vertonung einer Allegorie entsprechen müsste. Die Tätigkeit des Komponisten kann in dieser Hinsicht zwar 
aufgrund der Vorgaben durch den Librettisten nicht als vollkommen frei und willkürlich bezeichnet werden, ist aber wohl eine kreative, weil ihr Produkt, die Vertonung, auch neue Perspektiven auf den Text ermöglicht.

Die Gleichnisarie ist mit Blick auf den Rezipienten als ein Schauplatz semiotischer Lernprozesse betrachtet worden, wo doch die musikalischen Strukturen zu Beginn der Arienrezeption noch als asemantisch bzw. vage begriffen, zum Ende derselben aber als semantisiert gelten können. Es ging in der vorliegenden Untersuchung u. a. darum, die Mechanismen des werkinternen Zeichenlernens offenzulegen, die selbstverständlich auch dann wirksam sind, wenn die Arien nicht original für das jeweilige Werk komponiert, sondern früheren Werken entlehnt sind. Nicht nur aufgrund solcher Semioseprozesse kann die allegorische bzw. Gleichnisarie trotz der in der Operngeschichte immer wieder geäußerten Vorbehalte hinsichtlich ihrer dramaturgischen Funktion als ein wertvoller Untersuchungsgegenstand der Semiotik im Allgemeinen und der Polysemiotizitätsforschung im Speziellen entdeckt werden. Die systematische Erforschung eines größeren Arienkorpus ist jedenfalls ein weiterhin bestehendes Forschungsdesiderat.

\section{Endnoten}

1 An dieser Stelle lässt sich nur kurz anmerken, was im Rahmen des vorliegenden Aufsatzes nicht geleistet werden kann: Das Abfassen eines Librettos erfordert nicht nur Rücksichtnahmen auf die Bedürfnisse des Komponisten, sondern auch auf die des Regieteams und der Aufführenden. Denn auch sie profitieren bei ihren Interpretationen von der Anschaulichkeit der sprachlichen Vorlage.

2 ,Übersetzung' wird hier in einem weiten Sinne gebraucht, da aus heuristischen Gründen auch solche Phänomene metaphorisch als Übersetzung bezeichnet werden können, die über semiotische Grenzen hinaus verlaufen. Die Unterscheidung von ,intralingualem', interlingualem ' und ,intersemiotischem 'Übersetzen geht auf Jakobson (1974) zurück. Auch Bruni (1971, 271) sieht übrigens die Musik in einer Gleichnisarie als intersemiotische Übersetzung des Textes an, wenn er - ohne ein explizit übersetzungswissenschaftliches Interesse zu verfolgen - von einem „traslato musicale" spricht

$3 \mathrm{Zu}$ den Begriffen des ,polysemiotischen' Komplexes und der ,synsemiotischen' Relationen cf. Agnetta (2018) sowie ders. (2017: 1f.).

4 Auch in anderen polysemiotischen Kommunikaten fungieren Metaphern, Metonymien, Vergleiche und Phraseologismen häufig als Schnittstellen zwischen den unterschiedlichen Zeichensystemen. In Bezug auf Text-Bild-Kommunikate im Bereich der Anzeigenwerbung etwa hat Stöckl (2004, 150) aufgezeigt, dass es vor allem diese Sprachmittel sind, „die im Text als wichtige sprachliche Bezugspunkte für materielle Bilder fungieren. [...] Dass vorzugsweise Idiome mit materiellen Bildern interagieren, erklärt sich aus ihrer Eigenschaft, mit der wörtlichen (oder eben bildlichen) Bedeutung bzw. Lesart selbst über eine bildliche Grundlage zu verfügen und daher wie sprachlich generierte Bilder im Text funktionieren zu können“. 
5 Alle Aufführungshinweise in und Notenbeispiele aus den Arien folgen auch in der Taktangabe (,T.') der von Bizzarini/Borin edierten Griselda-Partitur (cf. Vivaldi 2015).

6 Diesem Urteil kann in dieser radikalen Pauschalität nicht beigepflichtet werden, wie u. a. Tegtmeyer (2016) in einer gut recherchierten synoptischen Studie systematisch dargelegt hat. Es ist allerdings wahr, dass die Sinn- (auf der parole-Ebene) und womöglich die Bedeutungskonstitution (langueEbene) in der Musik anders vonstattengeht als in der Sprache. Diesen Unterschieden kann aber im Folgenden nicht detailliert nachgegangen werden.

7 Für eine werkimmanente Konzeption des semantischen Potenzials musikalischer Strukturen spricht zudem die Tatsache, dass der Komponist ebenjene (und keine anderen) Mittel ausgewählt hat, um die Aussage des Librettotextes auszudeuten. Für ihn als Produzenten hat die Musik von vornherein die semantischen Dimensionen bzw. den Sinn, dessen Nachvollzug er sich vom Empfänger im Rezeptionsakt erhofft. Auch wenn dieser beispielsweise eine bereits existierende Arie aus einer anderen Oper parodiert, die u. U. einen ganz anderen Text aufwies und diese mit mehr oder weniger geringfügigen Änderungen an den Inhalt des neuen Textes anpasst, so wählt er dennoch in der Regel eine Arie aus, die zur Aussage dieser neuen Textgrundlage passt.

8 Dass musikalische Strukturen ihre endgültige Semantik erst im parole-Akt erhalten, haben diese übrigens, wie nicht zuletzt Mehltretter (2016: 27f.) beobachtet hat, mit der Metapher gemeinsam, denn wie die Musik erhält auch die Metapher erst in Interaktion in ihrem unmittelbaren Kontext eine vollständige und greifbare Bedeutung.

9 Dennoch schreibt auch Koch, dass die Musik eine ganz eigene Art hat, das Dichterwort auszudeuten und zu ergänzen: „Wenn die Tonkunst sich mit der Dichtung vereinigt, wenn, um durch ein Beyspiel zu erklären, der Dichter den Zustand seiner Seele, da er zwischen Furcht und Hoffnung schwebt, schildert, unter Donner und Blitz auf dem ungestümmen Meere im Nachen herumtreibt, und die Tonkunst unterstützt diese Bilder des Dichters, so weit es in ihrer Gewalt steht, durch ein gewisses Schwanken, durch ein gewisses ungestümmes anhaltendes Braußen, u. d. gl., so ist ihr Ausdruck lebendig. Und wer erkennet nicht aus diesem Beyspiele, daß auch in der Tonkunst der lebendige Ausdruck nicht die gewöhnliche Sprache, sondern eine besondere Art ist, und mit dem, was er in der Poesie ist, zum wenigsten die größte Aehnlichkeit hat?" (Koch 2007, 30; Hervorh. von M. A.)

10 Auch die von Wagner theoretisch umrissenen Mechanismen des werkinternen musikalischen Verweisens, die „Ahnung“ und die „Erinnerung“ (cf. Wagner 2008, 349ff.), sind Symptome einer solchen Emanzipation des musikalischen Zeichens.

\section{Bibliografie}

Agnetta, Marco: „Musik - Sprache - (Sprach-)Bild. Zur Semiotizität italienischer barocker Gleichnisarien (Teil 1)“. In: ATeM - Archiv für Textmusikforschung 2 (2017), 1-16, https://webapp. uibk.ac.at/ojs2/index.php/ATeM/article/view/1969/2017 (Zugriff 15.06.2018).

Agnetta, Marco: Ästhetische Polysemiotizität und Translation. Glucks Orfeo ed Euridice (1762) im italienischen, deutschen und französischen Kulturtransfer. Saarbrücken: universaar, 2018. 
Beaugrande, Robert-Alain de / Dressler, Wolfgang Ulrich: Einführung in die Textlinguistik. Tübingen: Max Niemeyer Verlag, 1981.

Bruni, Massimo: „Postille ed appunti sulla Griselda di Vivaldi“. In: Memorie e contributi alla musica dal medioevo all'età moderna offerti a F. Ghisi nel settantesimo compleanno (1901-1971). Bologna: A. M. I. S., 1971, 267-277.

Bucher, Hans-Jürgen: „Online-Interaktivität - Ein hybrider Begriff für eine hybride Kommunikationsform“. In: Bieber, Christoph / Leggewie, Claus (Hrsg.): Interaktivität: ein transdisziplinärer Schlïsselbegriff. Frankfurt a. M./New York: Campus, 2004, 132-167.

Cook, Nicholas: „Musikalische Bedeutung und Theorie“. Aus dem Englischen übersetzt von Alexander Becker. In: Becker, Alexander / Vogel, Matthias (Hrsg.): Musikalischer Sinn. Beiträge zu einer Philosophie der Musik. Frankfurt a. M.: Suhrkamp, 2007, 80-128.

Fricke, Harald: Gesetz und Freiheit. Eine Philosophie der Kunst. München: Beck, 2000.

Gier, Albert: „Perspektiven der Librettoforschung: die Gattung und ihre Theorie“. Vortrag am musikwissenschaftlichen Institut der Universität Bochum am 25. Juni 1998, http://opus4.kobv. de/opus4-bamberg/frontdoor/index/index/docId/7 (Zugriff 15.06.2018).

Harweg, Roland: „Prosa, Verse, Gesang. Zur Verbindung von Sprache und Musik“. In: Bernhart, Walter (Hrsg.): Die Semantik der musiko-literarischen Gattungen. Methodik und Analysen. Eine Festgabe für Ulrich Weisstein zum 65. Geburtstag. Tübingen: Gunter Narr Verlag, 1994, 135152.

Hesse, Horst-Peter: „Gehör. IV. Psychoakustische und psychophysikalische Grundlagen“. In: Finscher, Ludwig (Hg.): Die Musik in Geschichte und Gegenwart. Allgemeine Enzyklopädie der Musik begründet von Friedrich Blume. Zweite Ausgabe. Sachteil: Bd. 3. Kassel/Basel u. a.: Bärenreiter. Stuttgart/Weimar: Metzler, 1994ff., Sp. 1104-1118.

Istel, Edgar: Das Libretto. Wesen, Aufbau und Wirkung des Opernbuchs / nebst einer dramaturgischen Analyse des Librettos von Figaros Hochzeit. Berlin/Leipzig: Schuster \& Loeffler, 1914.

Jakobson, Roman: „Linguistische Aspekte der Übersetzung”. In: Jakobson, Roman (Hg.): Form und Sinn. München: Fink, 1974, 154-161.

Kneif, Tibor: „Musik und Zeichen. Aspekte einer nichtvorhandenen Semiotik“. In: Karbusicky, Vladimir (Hg.): Sinn und Bedeutung in der Musik. Texte zur Entwicklung des musik-semiotischen Denkens. Darmstadt: Wissenschaftliche Buchgesellschaft, 1990, 134-141.

Koch, Heinrich Christoph: Versuch einer Anleitung zur Composition. Hannover: Siebert, 2007.

Marx, Adolf B.: Gluck und die Oper. 2 Bde. Berlin: Janke, 1863.

Mehltretter, Florian: „Metaphoric Effects in a Monteverdi Madrigal. Intermediality and Relevance“. In: Mehltretter, Florian (Hg.): Wie semantisch ist die Musik? Beiträge zur Semiotik, Pragmatik und Ästhetik an der Schnittstelle von Musik und Text. Freiburg i. Br./Berlin/Wien: Rombach, 2016, 25-46.

Overbeck, Anja: Italienisch im Opernlibretto. Quantitative und qualitative Studien zu Lexik, Syntax und Stil. Berlin/Boston: de Gruyter, 2011.

Schering, Arnold: „Symbol in der Musik“. In: Karbusicky, Vladimir (Hg.): Sinn und Bedeutung in der Musik. Texte zur Entwicklung des musiksemiotischen Denkens. Darmstadt: Wissenschaftliche Buchgesellschaft, 1990, 37-46. 
Stöckl, Hartmut: Die Sprache Im Bild - Das Bild in Der Sprache. Zur Verknüpfung von Sprache und Bild im massenmedialen Text. Konzepte. Theorien. Analysemethoden. Berlin/Boston: de Gruyter, 2004.

Strohm, Reinhard: Die italienische Oper im 18. Jahrhundert. Wilhelmshaven: Heinrichshofen, 1979. Strohm, Reinhard: The Operas of Antonio Vivaldi. 2 Bde. Florenz: Olschki, 2008.

Tegtmeyer, Henning: „Pythagoräismus versus Psychologismus? Über falsche Gegensätze in der Musikphilosophie“. In: Mehltretter, Florian (Hg.): Wie semantisch ist die Musik? Beiträge zur Semiotik, Pragmatik und Ästhetik an der Schnittstelle von Musik und Text. Freiburg i. Br./Berlin/ Wien: Rombach, 2016, 11-23.

Vivaldi, Antonio: La Griselda, RV 718. Hg. Marco Bizzarini / Alessandro Borin. Mailand: Ricordi, 2015.

Wagner, Richard: Oper und Drama. Stuttgart: Reclam, 2008. 